Jan. 16, 2001

\title{
On a Classical, Geometric Origin of Magnetic Moments, Spin-Angular Momentum and the Dirac Gyromagnetic Ratio
}

\author{
Ezra T. Newman \\ Dept of Physics and Astronomy, University \\ of Pittsburgh, Pittsburgh PA 15260
}

\begin{abstract}
By treating the real Maxwell Field and real linearized Einstein equations as being imbedded in complex Minkowski space, one can interpret magnetic moments and spinangular momentum as arising from a charge and mass monopole source moving along a complex world line in the complex Minkowski space. In the circumstances where the complex center of mass world-line coincides with the complex center of charge world-line, the gyromagnetic ratio is that of the Dirac electron.
\end{abstract}




\section{Introduction}

Many years ago in a brief note concerning relativistic angular momentum, we made the claim, largely as an afterthought and with only a skimpy argument presented, that there was a simple geometric classical argument that led to the Dirac value of the gyromagnetic ratio of a charged massive particle. There was no mention of either quantum mechanics or the Dirac equation. The argument made then, was based on a kinematic analogy concerning certain algebraic properties of the angular momentum tensor and the electric-magnetic dipole tensor, and has on several occasions been reasonably questioned. In the present note we return to the same issue and give the complete argument, including details of the dynamics that lead to the relevant algebraic properties.

We make the following pair of claims: Consider first both the vacuum Maxwell field and the linearized vacuum gravitational field on (ordinary real) Minkowski space. It is possible to view (purely in a mathematical sense by the analytic continuation of the real fields into the complex) that the magnetic moment $\vec{\mu}$ of a particle with charge $q$, arises from the particle being displaced an imaginary distance $\left(i \vec{Y}_{e m}\right)$ into complex Minkowski space so that $\vec{\mu}=q \vec{Y}_{e m}$. Also, classical relativistic spin-angular momentum $\vec{S}$, can be interpreted (using linearized general relativity) as arising from the displacement $\left(i \vec{Y}_{g}\right)$ of a massive monopole particle, $m$, (Schwarzschild solution) also into the complex so that $\vec{S}=m c \vec{Y}_{g}$. We refer to $\vec{Y}_{e m}$ and $\vec{Y}_{g}$ as respectively, the complex centers of charge and mass. The second claim is simply the observation that when the two imaginary displacements are the same, i.e., $\vec{Y}_{e m}=\vec{Y}_{g}$, then the gyromagnetic ratio $|\mu| /|S|$ is the Dirac value, $q / m c$. This can be restated more geometrically as; given a real solution of the linearized Einstein equations (on real Minkowski space) with non-vanishing mass and a solution of the Maxwell equations with non-vanishing charge, if the associated angular momentum and magnetic moment are interpreted as arising from a complex center of mass and a complex center of charge that coincide, then the resulting gyromagnetic ration is that of Dirac. We stress that this is simply an observation and it remains to be shown if it has any further physical significance.

A point that we want to emphasis is that we are dealing, here, strictly with the vacuum fields and are not considering the nature or structure of the sources. What we are calling the charge, the dipole moments, the mass and angular momentum are the coefficients of the relevant terms in $r^{-1}$ expansions of the Maxwell and gravitational

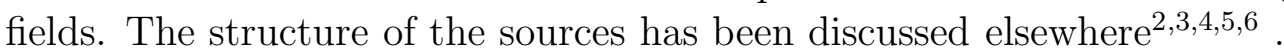

Another point that we wish to make is that this result, though it appears to depend on the linearized Einstein equations, remains though in a different form in full GR. The charged Kerr solution (with parameters $(m, q, Y)$ also has the Dirac gyromagnetic ratio and can be "obtained" from the Schwarzschild solution by a complex translation.2

In Sec.II, we will write the Maxwell equations in such a way so that the complex 
interpretation becomes obvious and furthermore show how the real simple Coulomb solution, by a complex translation, can be reinterpreted as having a magnetic moment. In Sec. III, we will rewrite the linearized vacuum Einstein equations as equations for the Weyl tensor (often considered as the gravitational analogue of the Maxwell tensor) and see its remarkable similarity with the Maxwell equations. The complex translation interpretation of angular momentum simply follows as does our conclusion concerning the gyromagnetic ratio. Finally, in Sec. IV, we point out possible physical consequences of these observations. In an appendix we examine, without a real resolution, how a rather strange complex coordinate transformation is equivalent to a real transformation.

\section{The Vacuum Maxwell Equations}

We begin with the standard vacuum Maxwell equations defined on Minkowski space,

$$
\begin{aligned}
\nabla_{a} F^{a b} & =0, \\
\nabla_{[a} F_{c d]} & =0 \Leftrightarrow \nabla_{a} F^{* a b}=0
\end{aligned}
$$

with

$$
\begin{aligned}
F^{* a b} & =\frac{1}{2} \eta^{a b c d} F_{c d}, \\
F^{* * a b} & =-F^{a b} .
\end{aligned}
$$

We use for the flat metric $\eta^{a b}=\operatorname{diag}(1,-1,-1,-1)$ and $\eta_{0123}=-\eta^{0123}=1$, with $\eta_{0 i j k}=\epsilon_{i j k}$ and $\eta^{0 i j k}=-\epsilon^{i j k}$.

By defining the (self-dual) complex combination

$$
\begin{aligned}
W^{a b} & \equiv F^{a b}-i F^{* a b}, \\
W^{* a b} & =F^{* a b}+i F^{a b}=i W^{a b} \\
W^{a b} & =-i W^{a b *},
\end{aligned}
$$

the Maxwell equations become the single complex equation

$$
\nabla_{a} W^{a b}=0 .
$$

Remark 1 It is often useful to see the matrix form of $W^{a b}$ in terms of Cartesian components of the electric and magnetic fields, i.e., as

$$
\begin{aligned}
& W^{a b}=F^{a b}-i F^{* a b}= \\
& \left(\begin{array}{llll}
0, & -\left(E^{1}+i B^{1}\right), & -\left(E^{2}+i B^{2}\right), & -\left(E^{3}+i B^{3}\right) \\
E^{1}+i B^{1}, & 0, & -i\left(E^{3}+i B^{3}\right), & i\left(E^{2}+i B^{2}\right) \\
E^{2}+i B^{2}, & i\left(E^{3}+i B^{3}\right), & 0, & -i\left(E^{1}+i B^{1}\right) \\
E^{3}+i B^{3}, & -i\left(E^{2}+i B^{2}\right), & i\left(E^{1}+i B^{1}\right), & 0
\end{array}\right)
\end{aligned}
$$


By decomposing Eq.(8), into its time-space parts, using $(i, j, k)$ for the space part and 0 for the time part and

$$
\begin{aligned}
W^{i j} & =i \epsilon^{i j k} W_{0 k}=-i \epsilon^{i j k} W^{0 k} \\
W^{i} & =W^{0 i}
\end{aligned}
$$

we have

$$
\begin{aligned}
\nabla_{0} W^{0 j}+i \epsilon^{j i k} \nabla_{i} W^{0 k} & =0 \\
\nabla_{i} W^{0 i} & =0
\end{aligned}
$$

In 3-vector terms, it becomes

$$
\begin{aligned}
\partial_{t} \vec{W}+i \operatorname{curl} \vec{W} & =0 \\
\operatorname{div} \vec{W} & =0
\end{aligned}
$$

the vector form of Maxwell's equations, with

$$
\vec{W}=\vec{E}+i \vec{B}
$$

The value in this formulation, is that we can now think of the equations, as equations for a complex vector on a four complex dimensional manifold, $M_{C}$, the complexification of Minkowski space, $M$.

When any complex solution is found as a function of the four complex coordinates,

$$
z^{a}=x^{a}+i y^{a}
$$

i.e., of the form,

$$
\vec{W}\left(x^{a}+i y^{a}\right)
$$

one can simply chose, $y^{a}=c^{a}$, as four real constants and then take the real and imaginary parts of $\vec{W}$ to construct an $\vec{E}$ and $\vec{B}$, i.e., a real Maxwell field on real $M$. Since the equations do not have any explicit coordinate dependence, solutions will go over to solutions if they are transformed by complex Poincare transformations. If the transformations were real then the resulting new solutions are completely equivalent to the untransformed solutions; but if the transformations are intrinsically complex (or imaginary) then the new real solutions are genuinely different from the starting solution.

We will illustrate this with the Coulomb solution (in the vacuum region) transformed by a complex translation and in the process show how the magnetic moment arises from the complex translation. 
Considering the Coulomb solution in any of the following forms

$$
\begin{aligned}
E^{i} & =q r^{-2} n^{i}, B^{i}=0 \Longleftrightarrow W^{i}=e r^{-2} n^{i} \\
F^{a b} & =\frac{2 q n^{[a} v^{b]}}{r^{2}}
\end{aligned}
$$

with a unit radial vector, $n^{i}=\frac{x^{i}}{r}$ and particle velocity $v^{\nu}=(1,0,0,0)$ given in the rest-frame, we study the "same solution" thought of as translated into the complex, i.e., we replace the real coordinates by the complex translated ones;

$$
x^{i} \rightarrow \widehat{x}^{i}=x^{i}-C^{i}
$$

with

$$
C^{i}=X^{i}+i Y^{i}
$$

a complex spatial translation. If we denote the complex translated quantities with a hat $\left(^{\wedge}\right)$ then, keeping only low powers of $\widehat{r}^{-1}$, we have

$$
\begin{aligned}
& \widehat{r}^{2}=\left(x^{i}-C^{i}\right)^{2}=x^{i 2}-2 x^{i} C^{i}+C^{i} \cdot C^{i}=r^{2}\left(1-2 n^{i} \frac{C^{i}}{r}+O\left(r^{-2}\right)\right) \\
& \widehat{r}=r\left(1-r^{-1} n \cdot C+. .\right) \\
& \widehat{r}^{-2}=r^{-2}\left(1+2 r^{-1} n \cdot C+\ldots\right) \\
& \quad \widehat{n}^{i}=\frac{\widehat{x}^{i}}{\widehat{r}}=\frac{x^{i}-C^{i}}{r\left(1-r^{-1} n \cdot C\right)}=\frac{\left(x^{i}-C^{i}\right)}{r}\left(1+r^{-1} n \cdot C \ldots\right)
\end{aligned}
$$

The new solution, obtained from the complex translated space coordinates, keeping terms up to $r^{-3}$, is

$$
\begin{aligned}
\widehat{W}^{i} & =q \widehat{r}^{-2} \widehat{n}^{i}, \\
\widehat{W}^{i} & =q r^{-2}\left(n^{i}-\frac{C^{i}}{r}\right)\left(1+2 r^{-1} n \cdot C\right)\left(1+r^{-1} n \cdot C\right), \\
\widehat{W}^{i} & =q r^{-2}\left\{n^{i}-\frac{C^{i}}{r}+3 n^{i} r^{-1} n \cdot C\right\} \\
\widehat{W}^{i} & =q r^{-2} n^{i}+r^{-3}\left\{3 n^{i} n \cdot D_{(C)}-D_{(C)}^{i}\right\}+O\left(r^{-4}\right)
\end{aligned}
$$

with the complex dipole moment $D_{(C)}^{i}$

$$
D_{(C)}^{i}=q C^{i} \text { and } n \cdot D_{(C)}=D_{(C)}^{i} n^{i} .
$$

This correspond\$ 8 to a static electric monopole and dipole field with, in addition, a magnetic dipole field. The real part of $D^{i}$ is the electric dipole moment, 


$$
\operatorname{Re} D^{i}=q X^{i}
$$

and the imaginary part is the magnetic dipole moment,

$$
\operatorname{Im} D^{i}=\mu^{i}=q Y^{i}
$$

both arising from the complex translation. Note that identifying the magnetic moment with $q Y^{i}$ is dimensionally correct.

The relativistic version of Eq.(22) is

$$
\widehat{W}^{a b}=2 q r^{-2} n^{[a} v^{b]}-r^{-3}\left\{2 n^{[a} v^{b]} 3 D_{(C)}^{a b} n_{a} v_{b}+D_{(C)}^{a b}\right\}
$$

with the complex dipole moment

$$
\begin{aligned}
& D_{(C)}^{a b}=2 q\left(X^{[a}+i Y^{[a}\right) v^{b]} \\
& X^{b} v_{b}=Y^{b} v_{b}=0
\end{aligned}
$$

and

$$
n \cdot D_{(C)}=-D_{(C)}^{a b} n_{a} v_{b}
$$

remembering that $n_{a} n_{b} \eta^{a b}=-1$.

If we began with simply a moving charge, $q$, with an electric dipole moment $D_{(e)}^{a}$ and magnetic dipole moment $D_{(m)}^{a}$, such that $D_{(m)}^{a} v_{a}=D_{(e)}^{a} v_{a}=0, v^{a}$ being the particle four-velocity a real dipole tensor can be defined by $D_{(R)}^{a b}=2 D_{(e)}^{[a} v^{b]}+$ $\eta^{a b c d} D_{(m) c} v_{d}$. Under a shift in origin, $\widehat{x}^{a}=x^{a}-X^{a}$, it transforms as

$$
D_{(R)}^{\prime a b}=D_{(R)}^{a b}-2 q X^{[a} v^{b]}
$$

so that the electric part of $D_{(R)}^{a b}$ can be transformed away, with arbitrary $\lambda$, by

$$
X^{a}=q^{-1} D_{(e)}^{a}+v^{a} \lambda .
$$

This defines the (real) center of charge world line.

In an analogous fashion, from the complex point of view, the entire complex dipole moment can be transformed away, (in the stationary case) by

$$
D_{(C)}^{\prime a b}=D_{(C)}^{a b}-2 q Z^{[a} v^{b]}=0
$$

with

$$
Z^{a}=C^{a}+v^{a} \lambda \equiv\left(X^{a}+i Y^{a}\right)+v^{a} \lambda,
$$

defining (with arbitrary complex $\lambda$ ) the complex center of charge world line. In the time dependent case neither dipole can be transformed way but one could still 
define the complex center of charge so that instantaneously in some frame it can be transformed away.

Viewed in this formal manner, we see that both an electric and magnetic dipole can be interpreted as arising from a charged particle (with no dipole moments) moving along a complex world line.

\section{Linearized General Relativity}

Though in general relativity, (GR), the most common point of view is to think of the metric tensor as the basic and physical field variable with other variables obtained from the metric, there are nevertheless good reasons to consider other variables as perhaps more fundamental. In particular, one often thinks of the metric as analogous the vector potential of Maxwell theory with the Weyl tensor the analogue of the Maxwell field. Without being ideological about it, we will see that, at least, in linear theory the analogy is remarkably accurate.

Before we consider the linearization of GR, several remarks about the Weyl tensor are useful. The Weyl tensor, $C_{a b c d}$, which is the trace-free part of the full curvature tensor is equal to the curvature tensor when the vacuum Einstein equations are satisfied - with no cosmological constant. Under these circumstance the Bianchi Identities

$$
R_{a b[c d: e]} \equiv 0
$$

become differential equation for the Weyl tensor,

$$
\nabla_{[e} C_{c d] a b}=0
$$

or

$$
\nabla_{c} C^{* c d a b}=0
$$

where the dual on the first pair is

$$
C^{* c d a b}=\frac{1}{2} \eta^{c d e f} C_{e f}^{a b} .
$$

Since dualing on the right pair yields 9

$$
C^{* c d * a b}=-C^{c d a b}
$$

Eq.(36) can be written as

$$
\nabla_{c} C^{c d a b}=0
$$

By combining them and relabeling indices, we obtain, using the complex Weyl tensor, our basic field, the self-dual Weyl tensor

$$
W^{a b c d}=C^{a b c d}-i C^{* a b c d}
$$


with its field equation

$$
\nabla_{a} W^{a b c d}=0
$$

Note that

$$
W^{a b c d *}=i W^{a b c d} \text {. }
$$

By linearized GR, we will mean Eq.(41), where the covariant derivative is taken with the flat metric connection. This is the first example of the analogy with Maxwell theory, via Eq.(8).

In analogy with the fact that all the components of $W^{a b}$ can be expressed in terms of $W^{0 i}$, we have the following identities coming from Eq.(42)

$$
\begin{aligned}
W_{e f}{ }^{i j} & =-i \eta^{i j k 0} W_{e f k 0}=-i \epsilon^{i j k} W_{e f k 0} \\
W_{l m}{ }^{i j} & =-i \epsilon^{i j k} W_{l m k 0}=i \epsilon^{i j k} \epsilon_{l m n} W_{0 k}{ }^{n 0} \\
W^{i j 0 k} & =-i \epsilon^{i j l} W^{0 k}{ }_{l 0}=i \epsilon^{i j l} W^{0 k l 0}
\end{aligned}
$$

which allows all components of $W^{a b c d}$ to be expressed in terms of

$$
Z^{i j}=W^{0 i 0 j}=C^{0 i 0 j}-i C^{* 0 i 0 j} .
$$

$Z^{i j}$ is a complex, symmetric, trace-free, 3x3, tensor that contains all the 10 components of the Weyl tensor.

Writing this out in $3+1$ notation, we first have

$$
\nabla_{0} W^{0 b c d}+\nabla_{i} W^{i b c d}=0
$$

which, with $b=j$ and $b=0$, further decomposes into

$$
\begin{aligned}
\nabla_{0} W^{0 j c d}+\nabla_{i} W^{i j c d} & =0 \\
\nabla_{i} W^{i 0 c d} & =0 .
\end{aligned}
$$

With $c d=0 k$ and $c d=k l$ we have

$$
\begin{aligned}
\nabla_{0} W^{0 j 0 k}+\nabla_{i} W^{i j 0 k} & =0 \\
\nabla_{0} W^{0 j k l}+\nabla_{i} W^{i j k l} & =0
\end{aligned}
$$

and

$$
\begin{aligned}
\nabla_{i} W^{i 0 j 0} & =0 \\
\nabla_{i} W^{i 0 j k} & =0 .
\end{aligned}
$$


Using the Identities, (43), the last equation from both previous sets follow from the first equation of the set.

We thus have that $\nabla_{a} W^{a b c d}=0$ is equivalent to

$$
\begin{aligned}
\nabla_{0} W^{0 i 0 k}+i \epsilon^{i j l} \nabla_{j} W^{0 l 0 k} & =0 \\
\nabla_{i} W^{i 0 j 0} & =0
\end{aligned}
$$

or, with $Z^{i j}=E^{i j}+i B^{i j}=W^{0 i 0 j}$ in dyadic form, $\overleftrightarrow{Z}=\overleftrightarrow{E}+i \overleftrightarrow{B}$

$$
\begin{aligned}
\partial_{t} \overleftrightarrow{Z}+i \cdot \operatorname{curl} \overleftrightarrow{Z} & =0 \\
\operatorname{div} \overleftrightarrow{Z} & =0
\end{aligned}
$$

These are the linearized Einstein Equations for the Weyl tensor. Note the extraordinary similarity in form, with the complex version of the Maxwell Equations, (11). Again, as in the Maxwell case, we can generate new solutions, by complex Poincare transformations, from old solutions or equivalently by finding complex solutions depending on the complex Minkowski coordinates, $z^{a}=x^{a}+i y^{a}$ and choosing $y^{a}$ as constants.

Remark 2 It has been well known for many years that Eq. (41) can be viewed as the linear Einstein equations. We, however, have not been able to find in the literature the particular form, 53), that so mimics the Maxwell equations. Though we would be surprised, it might well be new.

We apply this idea to the monopole (or linearized Schwarzschild solution) and obtain the linearized Kerr solution. Beginning with the monopole solution in any of the equivalent forms

$$
\begin{aligned}
W^{a c b d} & =3 m r^{-3}\left(v^{[a} n^{c]} v^{[b} n^{d]}+\frac{1}{3} 4 v^{[a} \eta^{c][d} v^{b]}\right), \\
Z^{i j} & =W^{0 i 0 j}=3 m r^{-3}\left(n^{i} n^{j}-\frac{1}{3} \delta^{i j}\right), \\
\Psi_{2} & =\frac{1}{2} Z^{i j} n^{i} n^{j}=m / r^{3},
\end{aligned}
$$

with mass $m$, velocity vector $v^{a}=\delta_{0}^{a}$ and unit radial vector $n^{i}=\frac{x^{i}}{r},\left(n^{a} n_{a}=-1\right)$ we apply the complex translation

$$
x^{i} \rightarrow \widehat{x}^{i}=x^{i}-C^{i}=C^{i}=x^{i}-\left(X^{i}+i Y^{i}\right) .
$$

The new solution is first written as 


$$
\widehat{Z}^{i j}=3 m \widehat{r}^{-3}\left(\widehat{n}^{i} \widehat{n}^{j}-\frac{1}{3} \delta^{i j}\right)
$$

with (ignoring terms of higher order in $r^{-1}$ )

$$
\begin{aligned}
\widehat{r}^{2} & =\widehat{x}^{i} \cdot \widehat{x}^{i}=\left(x^{i}-C^{i}\right)^{2}=x^{i} \cdot x^{i}-2 x^{i} \cdot C^{i}+C^{i} \cdot C^{i} \\
& =r^{2}-2 x^{i} C^{i}+C^{i 2}=r^{2}\left(1-2 r^{-1} n \cdot C\right) \\
\widehat{r} & =r\left(1-r^{-1} n \cdot C\right) \\
\widehat{r}^{-3} & =r^{-3}\left(1+3 r^{-1} n \cdot C\right)
\end{aligned}
$$

and

$$
\begin{aligned}
\widehat{n}^{i} & =\frac{\widehat{x}^{i}}{\widehat{r}} \\
\widehat{n}^{i} & =\widehat{r}^{-1}\left(x^{i}-C^{i}\right)=\left(1+r^{-1} \vec{n} \cdot \vec{C}+. .\right)\left(n^{i}-r^{-1} C^{i}\right) \\
\hat{n}^{i} & =n^{i}+n^{i} r^{-1} \vec{n} \cdot \vec{C}-r^{-1} C^{i}
\end{aligned}
$$

Substituting (58) and (57) into (56a), by expanding, collecting and keeping only terms linear in $r^{-1} C^{i}$, we have

$$
\begin{aligned}
\widehat{Z}^{i j}= & 3 m \widehat{r}^{-3}\left(\widehat{n}^{i} \widehat{n}^{j}-\frac{1}{3} \delta^{i j}\right) \\
= & 3 m r^{-3}\left(1+3 r^{-1} \vec{n} \cdot \vec{C}\right) \cdot \\
& \left\{\left(n^{i}+n^{i} r^{-1} \vec{n} \cdot \vec{C}-r^{-1} C^{i}\right)\left(n^{j}+n^{j} r^{-1} \vec{n} \cdot \vec{C}-r^{-1} C^{j}\right)\right. \\
& \left.-\frac{1}{3} \delta^{i j}\right\} \\
= & 3 m r^{-3}\left(n^{i} n^{j}-\frac{1}{3} \delta^{i j}\right) \\
& +3 m r^{-4}\left\{\vec{n} \cdot \vec{C}\left(5 n^{i} n^{j}-\delta^{i j}\right)-\left(n^{i} C^{j}+n^{j} C^{i}\right)\right\}
\end{aligned}
$$

or

$$
\begin{aligned}
\widehat{Z}^{i j}= & 3 m r^{-3}\left(n^{i} n^{j}-\frac{1}{3} \delta^{i j}\right) \\
& +3 r^{-4}\left\{\vec{n} \cdot \vec{D}_{(g)}\left(5 n^{i} n^{j}-\delta^{i j}\right)-\left(n^{i} D_{(g)}^{j}+n^{j} D_{(g)}^{i}\right)\right\}
\end{aligned}
$$

with $D_{(g)}^{k}=m C^{k}=m\left(X^{k}+i Y^{k}\right)$, the complex gravitational dipole moment. This result is the analogue of the Maxwell result, (22). $R e D_{(g)}^{k}=m X^{k}$ is the mass dipole moment and, as we will see shortly, $\operatorname{Im} D_{(g)}^{k}=m Y^{k}$ is the spin-angular momentum.

Remark 3 The quantity $\Psi_{2}=\frac{1}{2} \widehat{Z}^{i j} n^{i} n^{j}=m r^{-3}+3 r^{-4} \vec{n} \cdot \vec{D}_{(g)}$ obtained from (63) is the same as the linearized Kerr solution. In that case the Kerr parameter a, the angular momentum per unit mass, is the same as the magnitude of our $Y^{i}$. 
The relativistic version of $D_{(g)}^{k}$ is given by

$$
D_{(g)}^{a b}=2 D_{(g)}^{[a} v^{b]}
$$

with $v_{a}$ the particle velocity and

$$
\begin{aligned}
D_{(g)}^{a} & =D_{(g)}^{a b} v_{b}, \\
\vec{n} \cdot \vec{D}_{(g)} & =D_{(g)}^{a b} n_{b} v_{a} \\
D_{(g)}^{a} v_{a} & =0 .
\end{aligned}
$$

When $v_{a}=(1,0,0,0)$, then $D_{(g)}^{a}=\left(0, D_{(g)}^{k}\right)$.

Note that we can identify (and decompose) $D_{(g)}^{a b}$ with respect to a real tensor $M^{a b}$ (the angular momentum tensor) by

$$
D_{(g)}^{a b}=\left(M^{a b}-i M^{* a b}\right)
$$

where $M^{a b}$ can be further decomposed into

$$
M^{a b}=L^{a b}+S^{a b} .
$$

so that $L^{a b}$ (the orbital angular momentum tensor) is defined by

$$
L^{a b}=m 2 X^{[a} v^{b]}=2 X^{[a} p^{b]}
$$

with $X^{a}$ obtained from the $M^{a b}$ by

$$
m X^{a}=M^{a b} v_{b}=m^{-1} M^{a b} p_{b}
$$

and

$$
S^{* a b}=-2 S^{[a} v^{b]}
$$

where $S^{b}$, the scaled Pauli-Lubanski spin vector, is obtained from $M^{a b}$ by

$$
S^{b}=v_{a} M^{* a b}=m^{-1} p_{a} M^{* a b} .
$$

In the rest frame of the source, $v_{a}=(1,0,0,0)$, we have that

$$
\begin{aligned}
M^{a b} & =\left(\begin{array}{llll}
0, & -m X_{x}, & -m X_{y}, & -m X_{z} \\
m X_{x}, & 0, & -S_{z}, & S_{y} \\
m X_{y}, & S_{z}, & 0, & -S_{x} \\
m X_{z}, & -S_{y}, & S_{x}, & 0
\end{array}\right) \\
& =\left(\begin{array}{llll}
0, & -D_{x}, & -D_{y}, & -D_{z} \\
D_{x}, & 0, & -S_{z}, & S_{y} \\
D_{y}, & S_{z}, & 0, & -S_{x} \\
D_{z}, & -S_{y}, & S_{x}, & 0
\end{array}\right)
\end{aligned}
$$


with $D^{i}=m X^{i}$, so that

$$
\begin{aligned}
& \left(M^{a b}-i M^{* a b}\right) \\
= & \left(\begin{array}{llll}
0, & -\left(m X_{x}+i S_{x}\right), & -\left(m X_{y}+i S_{y}\right), & -\left(m X_{z}+i S_{z}\right) \\
m X_{x}+i S_{x}, & 0, & i\left(m X_{z}+i S_{z}\right), & -i\left(m X_{y}+i S_{y}\right) \\
m X_{y}+i S_{y}, & -i\left(m X_{z}+i S_{z}\right), & 0, & i\left(m X_{x}+i S_{x}\right) \\
m X_{z}+i S_{z}, & i\left(m X_{y}+i S_{y}\right), & -i\left(m X_{x}+i S_{x}\right), & 0
\end{array}\right) .
\end{aligned}
$$

Restoring the $c$, that has been tacitly taken as 1 , we have the dimensionally correct expression

$$
\left.\begin{array}{llll}
\left(M^{a b}-i M^{* a b}\right)= & \\
0, & -\left(m X_{x}+i c^{-1} S_{x}\right), & -\left(m X_{y}+i c^{-1} S_{y}\right), & -\left(m X_{z}+i c^{-1} S_{z}\right) \\
m X_{x}+i c^{-1} S_{x}, & 0, & i\left(m X_{z}+i c^{-1} S_{z}\right), & -i\left(m X_{y}+i c^{-1} S_{y}\right) \\
m X_{y}+i c^{-1} S_{y}, & -i\left(m X_{z}+i c^{-1} S_{z}\right), & 0, & i\left(m X_{x}+i c^{-1} S_{x}\right) \\
m X_{z}+i c^{-1} S_{z}, & i\left(m X_{y}+i c^{-1} S_{y}\right), & -i\left(m X_{x}+i c^{-1} S_{x}\right), & 0
\end{array}\right) .
$$

which, with (64) and (66), allows us to identify the spin angular momentum of the source with the complex displacement, i.e.,

$$
S^{k}=m c Y^{k}
$$

If, in Minkowski space, we have a massive charged particle at rest at the spatial origin, producing both an electric monopole field and mass monopole field and then (formally) consider this particle moved into the complex by an amount $C^{i}=i Y^{i}$, the new real solution has now both a magnetic dipole moment, (25), $\mu^{i}=q Y^{i}$ and a spin angular moment, $S^{i}=c m Y^{i}$. From this it follows that

$$
\mu^{i}=\frac{q}{m c} S^{i}
$$

and hence

$$
g_{e}=\frac{q}{m c}
$$

obtaining the Dirac value of the gyromagnetic ratio by a purely classical argument.

\section{Conclusions}

It has long been known, first pointed out by Brandon Carter, that the exact charged Ker 10 solution of the Einstein-Maxwell equations possessed the Dirac gyromagnetic ratio. Though at first this created a bit of a stir and interest, it nevertheless did not go anywhere and eventually faded from general interest. Nevertheless, in one form or another, intermittently there have been a long series of papers related to the issue. In some sense the present paper appears to give a simple geometric origin for Carter's observation. First it suggests that a particle's magnetic moment and intrinsic 
spin arises as a "shadow" or "projection" into the real, of a particle moving in the complex space-time and that in particular the Dirac value arises when a particle's complex center of charge coincides with its complex center of mass. One's first hope is that there is something profound about this result and perhaps there is, nevertheless after much thought, we and others have not seen anyway to further develop these ideas and at the present the issues lie dormant.11 Nevertheless, we are led to conjecture that every massive, charged elementary particle with spin will possess the Dirac gyromagnetic ratio.

As a final item, we point out that though the example used here was based on the static electric and mass monopole fields, these can be greatly generalized. One can construct real Maxwell and linearized Einstein fields by considering the generalization of the Lienard-Wiechart fields to that of a point source moving along an arbitrary complex world-line in complex Minkowski space. This leads to real fields with time varying magnetic dipoles and spin-angular momentum. The extension of these ideas to the full non-linear Einstein-Maxwell equations leads to algebraically special metrics.12 Considerable work is still required to fully understand them.

\section{Acknowledgments}

We sincerely thank both Gerry Kaiser and Andrzej Trautman for their questions, proddings, criticisms and insights that led to this work. This work was supported by the NSF under grant \#PHY-0088951. 


\section{Research Bibliography}

${ }^{1}$ E.T. Newman, J.Winicour, A Curiosity Concerning Angular Momentum, J. Math.Phys. 15, 1113, (1974)

${ }^{2}$ E.T. Newman, A. Janis, Note on the Kerr Spinning-Particle Metric. J. Math.Phys. 6, 915, (1965)

${ }^{3}$ W. Israel, Sources of the Kerr Metric, Phys. Rev. D 2,641 (1970)

${ }^{4}$ W. Israel, Line Sources in GR, Phys. Rev. D 15, 935 (1977) .

${ }^{5} \mathrm{H}$. Balasin, H. Nachbagauer, Distributional Energy-Momentum Tensor of the KerrNewman Spacetime Family, Class. Quantum Grav. 11, 1453, (1994)

${ }^{6}$ Gerald Kaiser, Distributional Sources for Newman's Holomorphic Field grqc/0108041,

${ }^{7}$ E.T. Newman, Maxwell Equations and Complex Minkowski Space, J.Math.Phys. 14, 102, (1973)

${ }^{8}$ L.Landau, E.M. Lifschitz, Classical Theory of Fields, Addison-Wesley, Reading, Mass, (1962)

${ }^{9}$ R. Penrose, W. Rindler, Spinors and Space-Time, Vol I , Cambridge University Press, Cambridge, (1984)

${ }^{10}$ E.T. Newman, E. Couch, K. Chinnapared, A. Exton, A. Prakash, R. Torrence, Metric of a Rotating Charged Mass, J.Math.Phys. 6, 918, (1965)

${ }^{11}$ A recent preprint by H. Pfister and M. King, A Rotating Charged Mass Shell: Dragging, Anti-Dragging and Gyromagnetic Ratio leads, from a totally different point of view, to a similar result. (submitted to Phys. Rev.D)

${ }^{12}$ Robert Lind, E.T. Newman, Complexification of the Algebraically Special Gravitational Fields, J.Math.Phys., 15, 1103, (1974)

${ }^{13}$ Morse and Feshbach, Methods of Theoretical Physics, Vol.II, p 1292, McGraw-Hill Book Co, USA (1953)

\section{Appendix}

Close 35 years ago we found a strange "derivation 2 " of the Kerr metric beginning with the Schwarzschild metric, by a rather mysterious process (trick!) involving complex transformations. \{The charged Kerr metric was first found, 10 from the ReisnerNordstrom metric, in this manner.\} Up to the present there does not yet appear to be a full rational explanation for the trick, except that it worked. Here we will give a 
partial explanation but confined to the flat-space limit. The explanation we offer is given via the complex translations, Eq.(16), that were discussed earlier.

First reviewing the process, we began with the Schwarzschild metric expressed in terms of a null tetrad, i.e.,

$$
g_{(S)}^{a b}=l^{a} n^{b}+n^{a} l^{b}-m^{a} \bar{m}^{b}-\bar{m}^{a} m^{b}
$$

with

$$
\begin{aligned}
l^{a} \partial_{a} & =\partial_{r} \\
n^{a} \partial_{a} & =\partial_{u}-\left(\frac{1}{2}-\frac{M}{r}\right) \partial_{r} \\
m^{a} \partial_{a} & =\frac{1}{\sqrt{2} r}\left(\partial_{\theta}+\frac{i}{\sin \theta} \partial_{\varphi}\right) .
\end{aligned}
$$

Then by considering $r$ to be complex, (i.e., $r \Rightarrow r_{c}$ ) and performing the complex coordinate transformation

$$
\begin{aligned}
r_{c} & =r^{*}+i a \cos \theta^{*} \\
u_{c} & =u^{*}-i a \cos \theta^{*} \\
\theta & =\theta^{*}, \quad \varphi=\varphi^{*}
\end{aligned}
$$

on the tetrad vectors, yielding $l^{* a} \partial_{a}, n^{* a} \partial_{a}, m^{* a} \partial_{a}$, and $\bar{m}^{* a} \partial_{a}$ (with $\bar{m}^{*}$ the complex conjugate of $m^{*}$ ) the new metric,

$$
g_{(K)}^{* a b}=l^{* a} n^{* b}+n^{* a} l^{* b}-m^{* a} \bar{m}^{* b}-\bar{m}^{* a} m^{* b}
$$

depending on two parameters, $(M, a)$, automatically satisfied the Einstein equations and was the known the Kerr metric. When the mass $(M)$ vanishes the metric is flat but is expressed in the unusual "Kerr coordinate system" and has the form;

$$
\begin{aligned}
d s^{2}= & d u^{* 2}+2 d u^{*} d r^{*}-2 a \sin ^{2} \theta^{*} d r^{*} d \varphi^{*} \\
& -\left(r^{* 2}+a^{2} \cos ^{2} \theta^{*}\right) d \theta^{* 2}-\left(a^{2}+r^{* 2}\right) \sin ^{2} \theta^{*} d \varphi^{* 2}
\end{aligned}
$$

or

$$
\begin{aligned}
d s^{2}= & d t^{* 2}-d r^{* 2}-2 a \sin ^{2} \theta^{*} d r^{*} d \varphi^{*} \\
& -\left(r^{* 2}+a^{2} \cos ^{2} \theta^{*}\right) d \theta^{* 2}-\left(a^{2}+r^{* 2}\right) \sin ^{2} \theta^{*} d \varphi^{* 2}
\end{aligned}
$$

We will be interested in this Kerr-form of the flat metric and try to see how the mysterious transformation, Eq.(78), that led to this form of the metric arises in a natural manner from the complex translations, Eq.16), in Minkowski space, 
described earlier in the text. The question we will answer is; how does the expression, (78),

$$
r_{c}=r^{*}+i a \cos \theta^{*}
$$

arise naturally and how is it used in a standard way to obtain Eq.(83)?

[The origin of the second of the complex transformations, Eq.(??), assuming the first, presents no problem since $t^{*}=t$, implies that, from $u_{c}=t-r$ and $u^{*}=t^{*}-r^{*}$ that

$$
\left.u_{c}=t-r=t^{*}-r^{*}-i a \cos \theta=u^{*}-i a \cos \theta^{*} .\right]
$$

We begin with the flat metric

$$
d s^{2}=d t^{2}-d r^{2}-r^{2}\left(d \theta^{2}+\sin ^{2} \theta d \varphi^{2}\right)
$$

and look for the ordinary real coordinate transformation to the Kerr-form, Eq.(83),

$$
\begin{aligned}
d s^{2}= & d t^{* 2}-d r^{* 2}-2 a \sin ^{2} \theta^{*} d r^{*} d \varphi^{*} \\
& -\left(a^{2} \cos ^{2} \theta^{* 2}+r^{* 2}\right) d \theta^{* 2}-\left(r^{* 2}+a^{2}\right) \sin ^{2} \theta^{*} d \varphi^{* 2} .
\end{aligned}
$$

From the complex translation,

$$
\begin{aligned}
\widehat{t} & =t, \quad \widehat{x}=x, \quad \widehat{y}=y, \\
\widehat{z} & =z-i a
\end{aligned}
$$

we have

$$
\begin{aligned}
r_{c}^{2} & \equiv \widehat{x}^{2}+\widehat{y}^{2}+\widehat{z}^{2}=x^{2}+y^{2}+z^{2}-a^{2}-2 i a z \\
r_{c}^{2} & =r^{2}-a^{2}-2 i a z=r^{2}-a^{2}-2 i a r \cos \theta \\
r^{2} & =x^{2}+y^{2}+z^{2}, \quad z=r \cos \theta .
\end{aligned}
$$

Since $r_{c}^{2}$ is complex we can write, with real $\left(r^{*}, q\right)$, by definition,

$$
r_{c}=r^{*}+i q,
$$

which when substituted into Eq.(91) yields

$$
r^{* 2}-q^{2}+2 i r^{*} q=r^{2}-a^{2}-2 i a r \cos \theta
$$

or

$$
\begin{aligned}
r^{2} & =r^{* 2}-q^{2}+a^{2} \\
\cos \theta & =-\frac{r^{*} q}{a \sqrt{r^{* 2}-q^{2}+a^{2}}}, \\
\varphi & =\varphi
\end{aligned}
$$


This is interpreted as the coordinate transformation between the polar coordinates $(r, \theta, \varphi)$ and $\left(r^{*}, q, \varphi\right)$ [which turns out to be a form of spheroidal coordinates ${ }^{13}$ ]. An alternate form of spheroidal coordinates arises when we take

$$
q=a \cos \theta^{*}
$$

so that

$$
r_{c}=r^{*}+i a \cos \theta^{*}
$$

and Eqs.(95) and (96) become

$$
\begin{aligned}
r^{2} & =r^{* 2}+a^{2} \sin ^{2} \theta^{*} \\
\cos \theta & =-\frac{r^{*} \cos \theta^{*}}{\sqrt{r^{* 2}+a^{2} \sin ^{2} \theta}}, \\
\varphi & =\varphi
\end{aligned}
$$

With this transformation the flat metric, Eq.(86), becomes

$$
\begin{aligned}
d s^{2}= & d t^{* 2}-\left(a^{2} \cos ^{2} \theta^{* 2}+r^{* 2}\right)\left(r^{* 2}+a^{2}\right)^{-1} d r^{* 2} \\
& -\left(a^{2} \cos ^{2} \theta^{* 2}+r^{* 2}\right) d \theta^{* 2}-\left(r^{* 2}+a^{2}\right) \sin ^{2} \theta^{*} d \varphi^{2} .
\end{aligned}
$$

Changing the coordinate $\varphi$ by

$$
\begin{aligned}
\varphi & =\varphi^{*}-\arctan \frac{r^{*}}{a} \\
d \varphi & =d \varphi^{*}-a\left(r^{* 2}+a^{2}\right)^{-1} d r^{*}
\end{aligned}
$$

leads directly to the flat "Kerr metric", Eq.(83);

$$
\begin{aligned}
d s^{2}= & d t^{2}-d r^{2}-2 a \sin ^{2} \theta^{*} d r d \varphi \\
& -\left(r^{* 2}+a^{2} \cos ^{2} \theta^{*}\right) d \theta^{* 2}-\left(a^{2}+r^{* 2}\right) \sin ^{2} \theta^{*} d \varphi^{2}
\end{aligned}
$$

obtained earlier by the "trick". Somehow the trick circumvents the hard detailed work of finding and doing the coordinate transformations. It automatically does the transformations, Eqs.(100),(101) and(105) and thus seems still to be rather mysterious even though it gives us how, $r_{c}=r^{*}+i a \cos \theta^{*}$, originates. 